June 13, 2018

\title{
Impulsive quantum measurements: restricted path integral versus von Neumann collapse
}

\author{
Tommaso Calarco \\ Dipartimento di Fisica, Università di Ferrara, \\ Via Paradiso 12, Ferrara, Italy 44100
}

(June 13, 2018)

\begin{abstract}
The relation between the restricted path integral approach to quantum measurement theory and the commonly accepted von Neumann wavefunction collapse postulate is presented. It is argued that in the limit of impulsive measurements the two approaches lead to the same predictions. The example of repeated impulsive quantum measurements of position performed on a harmonic oscillator is discussed in detail and the quantum nondemolition strategies are recovered in both the approaches.
\end{abstract}

03.65.Bz 


\section{INTRODUCTION}

Despite the impressive successes of quantum mechanics in explaining most experimental results about microscopic phenomena, a unique consistent quantum theory of measurement is still missing. In this field, an important achievement was made by von Neumann which postulated two ways for the evolution of the state vector: continuously, according to the linear Schrödinger equation, when time passes without a measurement on the system being performed; and discontinuously, according to probability laws, if a measurement is carried out (the so-called wavefunction collapse [1]). von Neumann first defined this feature "a peculiar dual nature of the quantum mechanical procedure, which could not be satisfactorily explained"円.

Mensky proposed [2] a phenomenological theory, based on Feynman path integral formalism [3], that expresses the a posteriori dynamical evolution of a system, undergoing a continuous measurement, in terms of the instrumental uncertainty and the output of the meter, supposed to be known before doing the calculations. The effect of the measurement is introduced in the space of the paths by means of an influence functional, which restricts the integration to those paths that lie around the measurement result. The restricted path integral approach has been applied to describe continuous [田] and impulsive [5] measurements in both linear and non-linear systems, to explain quantum Zeno effect [6] and to verify the possibility of testing quantum mechanics through temporal Bell-like inequalities in bistable potentials [7].

The most common objection raised against this approach is that it seems to treat the Feynman paths, in some sense, as real trajectories followed by the system. Mathematically, this means that the time evolution during the measurement is non-unitary, i.e. the wavefunction looses its normalization. In fact, it has been shown [4] that the obtained propagator is the same as the one associated to an effective Hamiltonian having a purely imaginary measurement term, which of course destroys the unitarity.

The aim of the present paper is to show how this effect is equivalent, in the impulsive limit, to von Neumann collapse. Section II is devoted to derive, from the restricted path integral propagator for a measurement of infinitesimal duration performed on a generalized coordinate, an analytic formula with which von Neumann formalism can be recovered. In Section III the calculation for a series of impulsive measurements in a generic potential with discrete energy levels, only requiring the knowledge of the energy eigenstates, is developed and is applied - in Section IV - to the case of repeated impulsive measurements of position in a harmonic oscillator [5]. Finally the predictions of Mensky and von Neumann theories are compared and conclusions are drawn in Section V.

1 "eine eigenartige Duplizität des Vorgehens, die nicht genügend erklärt werden könnte" [1] (p. 222 in the original version; p. 417 in the English translation). 


\section{QUANTUM MODEL FOR IMPULSIVE MEASUREMENTS OF POSITION}

The path integral formulation of quantum mechanics [3] provides a natural framework for handling continuous quantum measurements of position by restricting the integration, in the space of the trajectories $x(t)$ in the coordinate $x$ - assumed to be continuously monitored between times 0 and $\tau$-, to those paths which turn out to be compatible with the experimental outcome $a(t)$ within the instrumental error $\Delta a$ [2]. This can be done by means of a weight functional $w_{[a]}[x]$ depressing the contribution of paths whose distance from the $a(t)$ actually obtained (which is in general a continuous but not necessarily differentiable function of time) exceeds $\Delta a$. The propagator for the system is then written as a weighted integral:

$$
K_{[a]}\left(x^{\prime \prime}, \tau ; x^{\prime}, 0\right)=\int_{x(0) \equiv x^{\prime}}^{x(\tau) \equiv x^{\prime \prime}} d[x] \exp \left\{\frac{i}{\hbar} \int_{0}^{\tau} \mathcal{L}(x(t), \dot{x}(t), t) d t\right\} w_{[a]}[x]
$$

The probability distribution for the measurement output is a functional of $a(t)$; if $\psi(x, 0)$ is the wavefunction representing the initial state of the system, it has the form

$$
P_{[a]}=\frac{\left|\left\langle\psi_{[a]}(\tau) \mid \psi_{[a]}(\tau)\right\rangle\right|^{2}}{\int\left|\left\langle\psi_{[a]}(\tau) \mid \psi_{[a]}(\tau)\right\rangle\right|^{2} d[a]},
$$

where

$$
\psi_{[a]}\left(x^{\prime \prime}, \tau\right)=\int K_{[a]}\left(x^{\prime \prime}, \tau ; x^{\prime}, 0\right) \psi\left(x^{\prime}, 0\right) d x^{\prime} .
$$

Its dispersion estimates the actual experimental accuracy with which it is possible to extract the information on the position $x$, also called effective uncertainty:

$$
\Delta a_{\mathrm{eff}}^{2}=2 \int \frac{1}{\tau} \int_{0}^{\tau}[a(t)-\tilde{a}(t)]^{2} d t P_{[a]} d[a] .
$$

where $\tilde{a}(t)$ is the path which maximizes $P_{[a]}$. Of course, in general $\Delta a_{\mathrm{eff}} \geq \Delta a$.

The most natural way to represent in this framework an impulsive measurement at the initial instant is to consider it as the limit for infinitesimal time intervals of a continuous one with constant result $a(t) \equiv a$. Of course, in this case the probability distribution for the measurement results is a function of $a$.

Alternatively, one can take the limit directly in the path integral expression (11). A simple form for the weight functional is the Gaussian one [4]:

$$
w_{[a]}[x]=\exp \left\{-\kappa \int_{0}^{\tau}[x(t)-a(t)]^{2} d t\right\}
$$

where the measurement coupling $\kappa$ in general should be taken constant, in order to ensure that the dynamics can be described by a semigroup [2]. In the limit $\tau \rightarrow 0$, it turns out to be useful the position

$$
\kappa=\frac{1}{2 \Delta a^{2} \tau}
$$


in which $\Delta a$ assumes the proper meaning of a width in the space of paths. In this way, regardless of the form of the Lagrangian involved, one obtains an analytical expression [7]:

$$
\begin{aligned}
K_{a}\left(x^{\prime \prime}, x^{\prime}\right) & =\lim _{\tau \rightarrow 0} K_{[a(t) \equiv a]}\left(x^{\prime \prime}, \tau ; x^{\prime}, 0\right)= \\
& =\lim _{\tau \rightarrow 0} \int_{x(0) \equiv x^{\prime}}^{x(\tau) \equiv x^{\prime \prime}} d[x] \exp \left\{\int_{0}^{\tau}\left(\frac{i}{\hbar} \mathcal{L}-\frac{[x(t)-a]^{2}}{2 \Delta a^{2} \tau}\right) d t\right\}= \\
& =\lim _{\tau \rightarrow 0} \int_{x(0) \equiv x^{\prime}}^{x(\tau) \equiv x^{\prime \prime}} d[x] \exp \left\{-\frac{[x(0)-a]^{2} \pitchfork}{2 \Delta a^{2} \pitchfork}\right\}= \\
& =e^{-\frac{\left(x^{\prime}-a\right)^{2}}{2 \Delta a^{2}}} K\left(x^{\prime \prime}, 0 ; x^{\prime}, 0\right) \equiv \\
& \equiv e^{-\frac{\left(x^{\prime}-a\right)^{2}}{2 \Delta a^{2}}} \delta\left(x^{\prime \prime}-x^{\prime}\right) .
\end{aligned}
$$

The third line follows from neglecting, in the limit, the Lagrangian term with respect to the measurement term, going as $\tau^{-1}$ (this appears reasonable also from the physical point of view, because an impulsive measurement is assumed to induce a significant change in the state of the system during a negligible amount of time), and by applying the theorem of the mean value. $K$ is the propagator in the absence of measurement.

Let $\psi(x, t)$ be the wavefunction which describes a system undergoing an impulsive measurement at the instant $t$, with result $a$. From Eqn. (7) follows

$$
\psi_{a}\left(x, t^{+}\right)=w_{a}(x) \psi\left(x, t^{-}\right)
$$

where

$$
w_{a}(x) \stackrel{\text { def }}{=} e^{-\frac{(x-a)^{2}}{2 \Delta a^{2}}}
$$

Thus $\left\|\psi_{a}\left(t^{+}\right)\right\|$is the projection (i.e. the scalar product modulus) of $\psi\left(x, t^{-}\right)$on the weight function $w_{a}(x)$. So the quantity

$$
P(a)=\frac{\left\|\psi_{a}\left(t^{+}\right)\right\|^{2}}{\iint e^{-\frac{(x-a)^{2}}{\Delta a^{2}}}\left|\psi\left(x, t^{-}\right)\right|^{2} d x d a}=\frac{1}{\sqrt{\pi} \Delta a}\left\|\psi_{a}\left(t^{+}\right)\right\|^{2}
$$

represents - in analogy with Eqn. (2) - the probability that the system is found in the state described by $w_{a}(x)$.

It should be noted that in the limit $\tau \rightarrow 0$, if $\Delta a$ remains finite, $\kappa$ diverges as $\tau^{-1}$ : this means that von Neumann collapse is recovered by considering not only infinitesimal measurement durations, but also infinite coupling between the instrument and the system. Furthermore, in the limit of an extremely precise measurement $(\Delta a \rightarrow 0), \kappa$ diverges more rapidly than $\tau^{-1}$, and one easily obtains

$$
\lim _{\Delta a \rightarrow 0} P(a)=\left|\psi\left(a, t^{-}\right)\right|^{2}
$$

in agreement with the conventional interpretation of the wavefunction.

It is worth noting also that $\left\|\psi_{a}\left(t^{+}\right)\right\|^{2}$ can be less than 1: this follows from the nonunitarity of the temporal evolution induced in Eqn. (1) by the measurement term. Thus, in the impulsive limit, that apparently arbitrary modification of the dynamics of the system 
under monitoring is equivalent to the commonly accepted von Neumann postulate [四] of the discontinuity introduced by the measurement in the causal linear evolution of the state. For instance, the case of a perfect measurement which yields with certainty the information whether a particle is found within an interval of width $\Delta a$ around the position $a$, is recovered by choosing for the measurement operator the form, discontinuous and therefore less realistic than the (9),

$$
\hat{w}_{a}^{v . N .} \propto \theta(\hat{x}-[a-\Delta a]) \theta([a+\Delta a]-\hat{x}) .
$$

The restricted path integral approach to continuous measurements with a generic weight functional $w_{[a]}[x]$ appears therefore simply as a generalization of the idea of perfect instantaneous filtering in von Neumann theory of measurement being a smoothed version of it with finite accuracy and duration. The comparison between the results of the two approaches will be now performed in the case of stroboscopic measurements of position on a harmonic oscillator [5].

\section{STROBOSCOPIC MEASUREMENTS OF POSITION}

A stroboscopic sequence of measurements is obtained when an observable is monitored in an impulsive way at some definite instants, equally spaced by a quiescent time $\Delta T$ in which

no measurement is performed. Such a topic has been studied in detail [5] for characterizing Quantum Non Demolition [8] strategies for the measurement of the position of a quantum system. Particular advantages, in this field, can arise from applying the method developed in the preceding section and exploiting an energy eigenstates expansion, because of the uniformity to handle each form of the weight function $w_{a}(x)$, for instance the naïve one (12) - which expresses the usual representation of the measurement -, allowing analytical calculations for every potential [7]. For a generic system having discrete energetic levels (i.e. $\left.H|l\rangle=E_{l}|l\rangle\right)$, an initial state can be developed in energy eigenstates:

$$
\left|\psi\left(t_{0}\right)\right\rangle=\sum_{l=1}^{\infty} c_{l}|l\rangle
$$

If the coordinate of the system is measured with results $a_{n}, n=0,1, \ldots, N$ at each of the instants $t_{n} \equiv n \Delta T$, we get

$$
\left|\psi_{\left\{a_{n}\right\}_{n=0, \ldots, N}}\left(t_{N}^{+}\right)\right\rangle=\hat{w}_{a_{N}}\left(\prod_{j=1}^{N} e^{-\frac{i}{\hbar} \hat{H} \Delta T} \hat{w}_{a_{N-j}}\right)\left|\psi\left(t_{0}^{-}\right)\right\rangle,
$$

where $\hat{w}_{a}$ is the multiplication operator corresponding to the weight function (9). The normalization constants relative to each measurement are factorized and therefore can be neglected in the calculations: because they simplify in the definition (2) of $P(a), \Delta a_{\text {eff }}$ will not depend on them.

By considering Eqn. (13) and by inserting a completeness $\mathbf{1} \equiv \sum_{m=1}^{\infty}|m\rangle\langle m|$, Eqn. (14) is rewritten 


$$
\begin{aligned}
\left|\psi_{\left\{a_{n}\right\}_{n=0, \ldots, N}}\left(t_{N}^{+}\right)\right\rangle & =\sum_{l, m=1}^{\infty} c_{l}|m\rangle\left\langle m\left|\hat{w}_{a_{N}} e^{-\frac{i}{\hbar} \hat{H} \Delta T} \hat{w}_{a_{N-1}} \cdots \hat{w}_{a_{1}} e^{-\frac{i}{\hbar} \hat{H} \Delta T} \hat{w}_{a_{0}}\right| l\right\rangle= \\
& =\sum_{m=1}^{\infty}\left(\sum_{l=1}^{\infty} B_{m l}^{N} c_{l}\right)|m\rangle,
\end{aligned}
$$

where

$$
B_{m l}^{N}\left(\Delta T, \Delta a,\left\{a_{n}\right\}\right) \stackrel{\text { def }}{=}\left\langle m\left|\hat{w}_{a_{N}}\left(\prod_{j=1}^{N} e^{-\frac{i}{\hbar} \hat{H} \Delta T} \hat{w}_{a_{N-j}}\right)\right| l\right\rangle .
$$

By inserting $N$ times the identity operator $\mathbf{1} \equiv \sum_{n_{i}=1}^{\infty}\left|n_{i}\right\rangle\left\langle n_{i}\right|(i=1,2, \ldots, N)$, one gets

$$
B_{m l}^{N}=\sum_{n_{1}, n_{2}, \ldots, n_{N}=1}^{\infty} W_{m n_{1}}^{a_{N}}\left(\prod_{j=1}^{N-1} W_{n_{j} n_{j+1}}^{a_{N-j}}\right) W_{n_{N} l}^{a_{0}} \exp \left\{-\frac{i \Delta T}{\hbar} \sum_{i=1}^{N} E_{n_{i}}\right\}
$$

with

$$
W_{i j}^{a_{n}}(\Delta a) \stackrel{\text { def }}{\equiv} \int_{-\infty}^{+\infty} u_{i}^{*}(x) w_{a_{n}}(x) u_{j}(x) d x
$$

in which the $u_{i}(x) \equiv\langle x \mid i\rangle$ are the energy eigenfunctions in $\{\mathcal{X}\}$-representation.

Let $P_{a_{0}, a_{1}, \ldots, a_{N-1}}(a)$ be the probability that the $N^{\text {th }}$ measurement will give result $a$, when the results of all the previous measurements are known. Its dispersion can be now evaluated, in analogy with Eqn. (4), from Eqn. (15) through Eqs. (17)-(18). It is no more necessary to take the mean value on the measurement time as in (身), since $\tau \rightarrow 0$. The final expression is [7]:

$$
\Delta a_{\mathrm{eff}}^{2}\left(\left\{a_{n}\right\}_{n \leq N-1}, N\right)=2 \int_{-\infty}^{+\infty}\left(a-\tilde{a}_{N}\right)^{2}\left(\sum_{m=1}^{\infty}\left|\sum_{l=1}^{\infty} B_{m l}^{N}\left(a_{0}, \ldots, a_{N-1}, a\right) c_{l}\right|^{2}\right)^{2} d a
$$

where $\tilde{a}_{N}$ is the most probable result of the $N^{\text {th }}$ measurement, and the explicit dependences of $\Delta a_{\text {eff }}$ and of $B_{m l}^{N}$ from $\Delta T$ and from $\Delta a$ have been omitted.

Summarizing, once eigenvalues and eigenstates of a generic quantum system with discrete energy spectrum are known, from the decomposition in eigenstates of the initial wavefunction it is possible in principle to infer directly the value of the effective uncertainty after an arbitrary sequence of stroboscopic measurements. The actual calculation is difficult due to the presence of multiple sums on the numerable ensemble of the energy eigenstates. A practical evaluation requires to approximate the sums and the integrals in Eqn. (19), by truncating them to finite values, say respectively $N_{\text {MAX }}$ and $\pm A_{\text {MAX }}$. The accuracy of such an approximation has to be checked for comparison with results already known by other methods, both analytical (if any) or numerical.

\section{OPTIMAL MEASUREMENTS OF POSITION FOR A HARMONIC OSCILLATOR}

The harmonic potential shows its great importance in many fundamental problems in physics. In particular, it has been studied in the limit of infinite coupling for impulsive 
measurements, to search for Quantum Non Demolition (QND) observables [8]. These last are relevant for the detection of small displacements in the quantum limit of sensitivity for mechanical resonators used as gravitational wave antennas. In the present framework this problem can be formulated by noting that in general the effective uncertainty $\Delta a_{\text {eff }}$ is greater than the instrumental error $\Delta a$, expressing the spreading of the paths due to the effect of the back-action of the meter on the measured system. However for certain observables - namely the QND ones -, the ratio of the two uncertainties can be reduced to unity by applying optimal measurement strategies, without violating the Heisenberg principle [5]. In this section the method described before will be applied to the characterization of such QND strategies for a harmonic oscillator. The results obtained through the two measurement theories under consideration, expressed respectively by the weight functionals (5) and (9), will finally be compared.

For a harmonic oscillator, described by a Lagrangian of the form $\mathcal{L}=\frac{1}{2} m \dot{x}^{2}-\frac{1}{2} m \omega^{2} x^{2}$, it is possible to characterize also a priori the QND strategies for stroboscopic measurements of position, by means of the commutation relation of the observable $\hat{x}_{\mathrm{H}}-$ in the Heisenberg picture - at different times [8]:

$$
\left[\hat{x}_{\mathrm{H}}(t), \hat{x}_{\mathrm{H}}(t+\Delta T)\right]=\frac{i \hbar}{m \omega} \sin (\omega \Delta T) .
$$

From Eqn. (20) follows that a series of impulsive measurements of position with infinite precision, performed every $T / 2$ - where $T=2 \pi / \omega$ is the oscillation period -, will give perfectly predictable outcomes. Therefore one expects that, for impulsive measurements spaced in such a way, the inequality $\Delta a_{\text {eff }} \geq \Delta a$ could be saturated. This can be seen also by looking to the periodical wavepacket reformation via causal dynamical evolution. In the absence of measurements, the wavefunction $\psi\left(x, t_{0}\right) \equiv\left\langle x \mid \psi\left(t_{0}\right)\right\rangle$ of the state (13) will be, after half an oscillation period - in the same notations as above, specialized to the harmonic potential -,

$$
\begin{aligned}
\psi\left(x, t=t_{0}+\frac{T}{2}\right) & =e^{-\frac{i}{\hbar} \hat{H}\left(t-t_{0}\right)} \sum_{l=1}^{\infty} c_{l} u_{l}(x)= \\
& =\sum_{l=1}^{\infty} e^{-\frac{i}{\hbar}\left(l+\frac{1}{2}\right) \hbar \omega \frac{T}{2}} c_{l} u_{l}(x)= \\
& =e^{-i \frac{\pi}{2}} \sum_{l=1}^{\infty} e^{-i l \pi} c_{l}(-1)^{l} u_{l}(-x)= \\
& =-i \psi\left(-x, t_{0}\right),
\end{aligned}
$$

the third line following from the definition of $T$ and from the symmetry properties of the energy eigenfunctions.

Thus, every half-period, the wavepacket is reformed symmetrically with respect to the equilibrium position, except for an irrelevant phase factor. This means that, if $\psi\left(x, t_{0}\right)$ represented the state after an impulsive, infinite-precision $(\Delta a \rightarrow 0)$ measurement with outcome $a_{0}$, another measurement at $t=t_{0}+T / 2$ will yield with certainty the result $-a_{0} ;$ at $t=t_{0}+T$ this will be again $a_{0}$. Therefore, in the case $a_{0}=0$ [5], the optimal QND strategy - in which $\Delta a_{\text {eff }} \rightarrow \Delta a$, also for $\Delta a>0$ - is obtained by choosing the quiescent time $\Delta T=T / 2$. On the other hand, if $a_{0} \neq 0$, the optimality is reached either with $\Delta T=T / 2$ and alternated 
results $a_{n}=(-1)^{n} a_{0}(n=0,1,2, \ldots)$, or with constant results $a_{n} \equiv a_{0}$ but $\Delta T=T$.

Indeed, this behavior has been already obtained [5] by simulations based on the restricted path integral; here it will be recovered via von Neumann collapse, by means of the calculation described in the previous section. A preliminar comparison of it with the results of Ref. [5] should allow to test the degree of accuracy of the adopted approximations. To do this the conditions chosen there have been exactly replied: a Gaussian initial state with width $\sigma=5$ (in the unit system in which $\hbar=2 m=1$ ) centered at the origin, an instrumental error $\Delta a=1$ and a measurement time $\tau \simeq 10^{-5} T$. The investigation has been restricted to sequences of measurements with constant result $a_{n} \equiv a_{0}$ for $n \leq N-1$. In any symmetrical single-well potential, a symmetrical state localized in the middle of the well will change its width during the dynamical evolution but will remain centered in the same position at all times. Therefore, starting from such an initial state and choosing $a_{0}=0$, the most probable results of a measurement is still $\tilde{a}_{n} \equiv 0$ for every $n$ and any $\Delta T$. With these values of the parameters, the calculation has been repeated, varying the value of $\Delta T$, according to three methods:

$\mathcal{A}$ ) If an impulsive measurement is simulated [5] by means of a continuous one of short duration $\tau \ll \tau_{c} \stackrel{\text { def }}{=}(m / \hbar)\left(\Delta a^{-2}+\sigma^{-2}\right)^{-1}$, the computed quantities do not depend upon the duration of the measurement. With the choices made above, during the measurement the state remains a Gaussian one, having width

$$
\sigma(t)=-\frac{1}{4}\left[\alpha+\frac{\beta^{2}}{4\left(\frac{1}{2 \sigma^{2}}-\alpha\right)}\right]^{-2},
$$

with

$$
\alpha=\frac{i m \omega_{r} \cos \omega_{r} \tau}{2 \hbar \sin \omega_{r} \tau}, \quad \beta=-\frac{i m \omega_{r}}{\hbar} \sin \omega_{r} \tau, \quad \omega_{r}^{2} \equiv \omega^{2}-\frac{i \hbar}{\tau m \Delta a^{2}}
$$

After the end of the measurement, the state evolves causally, still preserving the Gaussian form with

$$
\sigma(t+\tau)=-\frac{1}{4}\left[\alpha^{\prime}+\frac{\beta^{\prime 2}}{4\left(\frac{1}{2 \sigma^{2}}-\alpha^{\prime}\right)}\right]^{-2}
$$

the coefficients refer now to the unmeasured dynamics:

$$
\alpha^{\prime}=\frac{i m \omega \cos \omega t}{2 \hbar \sin \omega t}, \quad \beta^{\prime}=-\frac{i m \omega}{\hbar} \sin \omega t
$$

By alternating these two types of evolution and by iterating this procedure $N$ times, one can obtain the width $\sigma_{N}$ of the state after $N$ stroboscopic measurements. In the impulsive regime, $\Delta a_{\text {eff }}$ is then easily calculated, according to its physical meaning [5]:

$$
\lim _{\tau \rightarrow 0} \Delta a_{\mathrm{eff}}(\tau)=\sqrt{\Delta a^{2}+\sigma_{N}^{2}}
$$


$\mathcal{B})$ As shown in Ref. 沺, the evaluation of the path integral (1) can be overcome by writing an effective Schrödinger equation which takes into account the effect of the measurement through an imaginary potential term:

$$
i \hbar \frac{\partial}{\partial t} \psi(x, t)=\left\{-\frac{\hbar^{2}}{2 m} \frac{\partial^{2}}{\partial x^{2}}+V(x)+\frac{i \hbar}{\tau \Delta a^{2}}[x-a(t)]^{2}\right\} \psi(x, t) .
$$

Eqn. (27) can be resolved with usual numerical techniques [9] on a space-time lattice. By turning on and off the measurement potential with periodicity $\Delta T$, one can compute the values of $\psi(x)$ on the chosen lattice after the desired measuring sequence; $\Delta a_{\mathrm{eff}}$ is then calculated via Eqs. (22) and (困).

$\mathcal{C})$ Method $\mathcal{A}$ applies only to Gaussian weight functionals. On the other hand, Eqn. (19) do not need this requirement; furthermore, it gives directly the value of $\Delta a_{\mathrm{eff}}$ after an arbitrary series of measurements, provided that convenient approximations have been made. The results shown in this paper have been obtained by restricting the extremes of integration in Eqn. (19) to $\pm 10 \Delta a_{\text {eff }}$, and by truncating the sums to $N_{\mathrm{MAX}}=20$. After comparison with the other approaches, this method will be applied to forms of the weight function different from the Gaussian one (9).

Fig. 1 shows the typical evolution of the dispersion $\Delta a_{\text {eff }}$ of the probability $P(a)$, as a consequence of repeated measurements with different periodicity (the cases of $\Delta T / T=\frac{1}{4}, \frac{1}{2}$ and $\frac{3}{4}$ are shown). Suddenly after the first measurement, performed at the initial instant of time, $\Delta a_{\text {eff }}$ approximates the width of the initial state; to be more precise, its value is given by Eqn. (26): in the considered case it is $\Delta a_{\text {eff }} \simeq 5.099$, as evidenced by the point $n=1$ of the graph. Due to the following measurements, the effective uncertainty reaches an asymptotic value $\Delta a_{\mathrm{eff}}^{a s}$ that depends on the quiescent time $\Delta T$. The values of $\Delta a_{\mathrm{eff}}^{a s}$ given by the different methods of calculation show a good agreement: so the more flexible approach $\mathcal{C}$ can be held for tested and can finally be applied to the comparison between the two measurement theories of Mensky and of von Neumann, which is the aim of the present paper. This is shown in the last figures.

Fig. 2 depicts the dependence of the asymptotic probability distribution for the measurement outcomes, $P_{a s}(a)$, on the quiescent time. In other words, it is considered the probability distribution for the result $a$ of the $N^{\text {th }}$ measurement $(N=16$ has been chosen, i.e. in the asymptotic region as shown in Fig. 1): in the graph are visualized together many curves relative to different sequences of measurements, each one characterized by a quiescent time $\Delta T$, as written on the $y$-axis. It can be seen with great evidence how the cases with $\Delta T$ integer or semi-integer multiple of the oscillation period are characterized by minimal dispersion in the $P_{a s}(a)$.

These results are summarized in Fig. 3, which shows the behavior of the asymptotic $\Delta a_{\text {eff }}$ versus $\Delta T$. It is obtained a periodic behavior, with minima each $\frac{T}{2}$ in which $\Delta a_{\text {eff }}^{a s}$ approximates the value $\Delta a$, which identify the optimal QND strategies. The same pattern would be obtained by choosing $a_{0} \neq 0$, although in this case, in general, one has also $\tilde{a}_{n} \neq a_{0}$ unless $\Delta T / T=i \in \mathbf{N}$. As explicitly shown above, the latter condition follows from the fact that, starting from a state not centered in the origin, one must wait at least an oscillation period for the wavepacket to be reformed at the same position. Thus the definition of $\Delta a_{\text {eff }}$ is no 
longer the same for all $\Delta T$, except for $\Delta T=T, 2 T, \ldots$ : for these quiescent times the QND strategies are recovered.

Finally, the basical aim of Fig. 3 is the comparison between the results obtained when the measurement is simulated respectively by a Gaussian weight (as for Fig. 2) or by a doublestep perfect filter as $\hat{w}_{a}^{v . N .}$ (12). The most significant difference between the two profiles consists in the presence, in the case $\grave{a}$ la von Neumann, of oscillations and flexes, probably attributable to the discontinuity of $w_{a}^{v . N .}(x)$. But the essential qualitative features (i.e. the ones that can find experimental application [ [ ] ) does not depend upon the detailed choice of the weight $w_{a}$. Thus, for impulsive measurements, von Neumann wavefunction collapse is simply a particular case of the restricted path integral theory.

\section{CONCLUSIONS}

The restricted path integral approach to quantum measurement can be straightforwardly specialized to impulsive measurements, providing analytical formulae for the evolution of the measured system. The model leads to a filtered state centered around the measurement result through instantaneous collapse of the wavefunction, having as width the instrumental error. Von Neumann ideal collapse is recovered by choosing a double-step ideal filter as measurement weight functional. The probability distribution of the possible measurement outputs has a dispersion whose behavior does not depend upon the form of the filter. In particular, for stroboscopic measurements of position on a harmonic oscillator, the optimal measuring strategies can be characterized both using an ideal or a Gaussian filter. On the other hand a continuous, smooth weight appears more adequate to represent in simple terms a physical process as the measurement. Indeed, the instrument being constituted by a collection of microscopic objects immersed in a thermodynamical environment [10], it is more likely to give rise to a Gaussian probability distribution for the measured physical quantities. A Gaussian weight is also favorable from the analytical point of view, and guarantees some natural group properties of the evolution under continuous monitoring [2].

Summarizing the obtained results, the restricted path integral theory of quantum measurement reveals to be a simple and natural extension of the naïve von Neumann instantaneous collapse theory to the more realistic case of continuous and non-ideal measurements, and appears as a more subtle tool for handling new fundamental problems (see for instance Refs. [5 (7)).

\section{ACKNOWLEDGMENTS}

I am grateful to R. Onofrio for encouraging me to write this paper, and to him and C. Presilla for a critical reading of the manuscript. 


\section{REFERENCES}

[1] J. V. von Neumann, Mathematische Grundlagen der Quantenmechanik, (Springer, Berlin, 1932); English translation by R. T. Beyer, Mathematical Foundations of Quantum Mechanics (Princeton University Press, Princeton, 1955).

[2] M. B. Mensky, Continuous Quantum Measurements and Path Integrals (IOP Publishers, Bristol and Philadelphia, 1993).

[3] R. P. Feynman and H. R. Hibbs, Quantum Mechanics and Path Integrals (McGraw-Hill, New York, 1955).

[4] M. B. Mensky, R. Onofrio, and C. Presilla, Phys. Lett. A161, 236 (1991).

[5] M. B. Mensky, R. Onofrio, and C. Presilla, Phys. Rev. Lett. 70, 2828 (1993).

[6] R. Onofrio, C. Presilla, and U. Tambini, Phys. Lett. A183, 135 (1993); U. Tambini, C. Presilla, and R. Onofrio, Phys. Rev. A51, 967 (1995).

[7] T. Calarco, R. Onofrio, Macrorealism, Non-Invasivity and Quantum Mechanics: a quantitative approach, in Proc. Conference on Foundations of Quantum Mechanics, Lecce 1993, (Kluwer, Dordrecht, 1994); Phys. Lett. A198, 279 (1995).

[8] C. V. Caves, K. S. Thorne, R. W. Drever, V. Sandberg, and M. Zimmermann, Rev. Mod. Phys. 52, 341 (1980).

[9] W. H. Press, Numerical Recipes: the Art of Scientific Computing (Cambridge University Press, Cambridge, 1986).

[10] A. Konetchnyi, M. B. Mensky, V. Namiot, Phys. Lett. A177, 283 (1993). 


\section{FIGURES}

FIG. 1. Evolution of $\Delta a_{\text {eff }}$ versus the number of measurements $n$, for some values of the quiescent time $\Delta T$. The continuous line refers to the analytical calculation done in Ref. [5] (crosses are numerical results) by approximating each impulsive measurement with a continuous one of short duration $\tau \simeq 10^{-5} T$. The dotted line is deduced from Eqn. (19), i.e. in the limit for $\tau \rightarrow 0$. The agreement, besides slight deviations in the initial transient, is within $1 \%$ in the asymptotic region.

FIG. 2. Asymptotic probability distribution $P_{a s}(a)$ for the measurement outcomes $a$ as function of the quiescent time $\Delta T / T$. The curve reaches its maxima (and correspondingly the minimum dispersion in $a$ ) at $\Delta T$ multiples of half an oscillation period. On top of the graph is superimposed a contour plot which shows the extremal regions.

FIG. 3. Normalized asymptotic effective uncertainty $\Delta a_{\text {eff }}^{a s} / \Delta a$ versus the quiescent time $\Delta T / T$ for stroboscopic measurements performed with Gaussian (5) or perfect (9) filtering. The curves does not differ qualitatively and coincide at the minima, as theoretically expected [5]. 


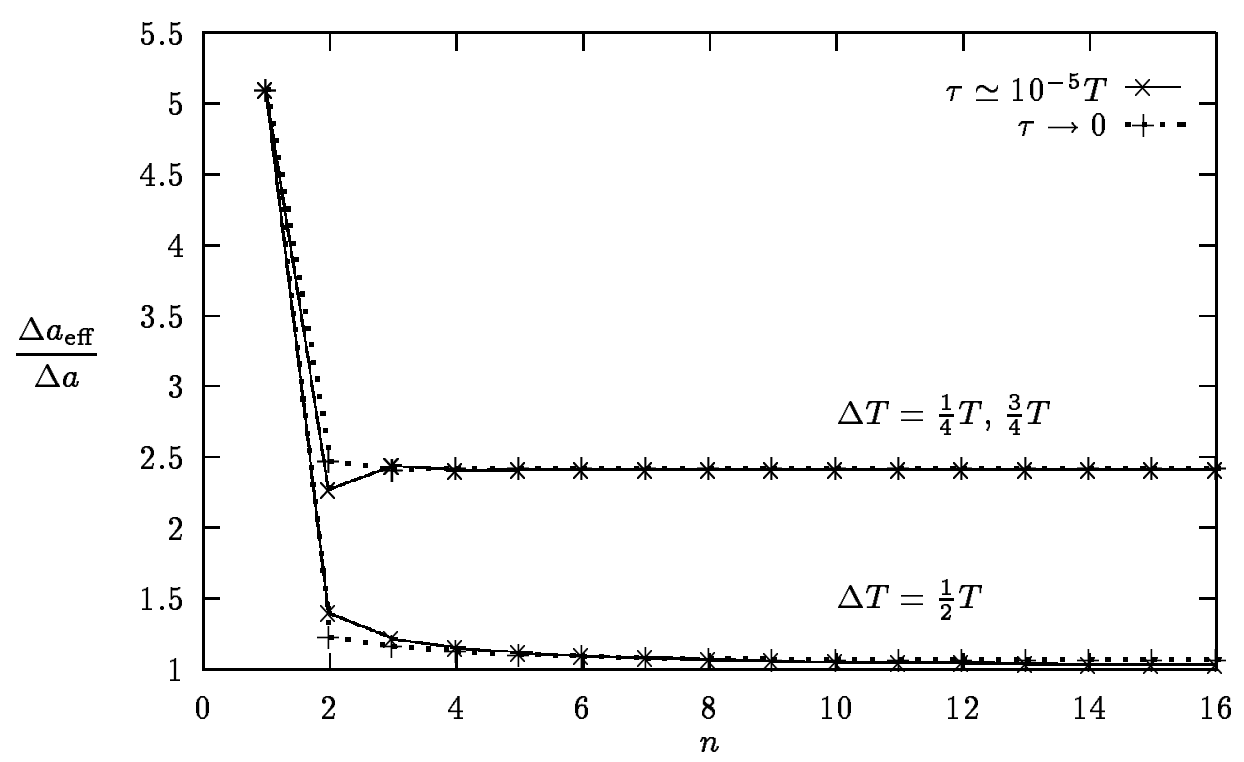




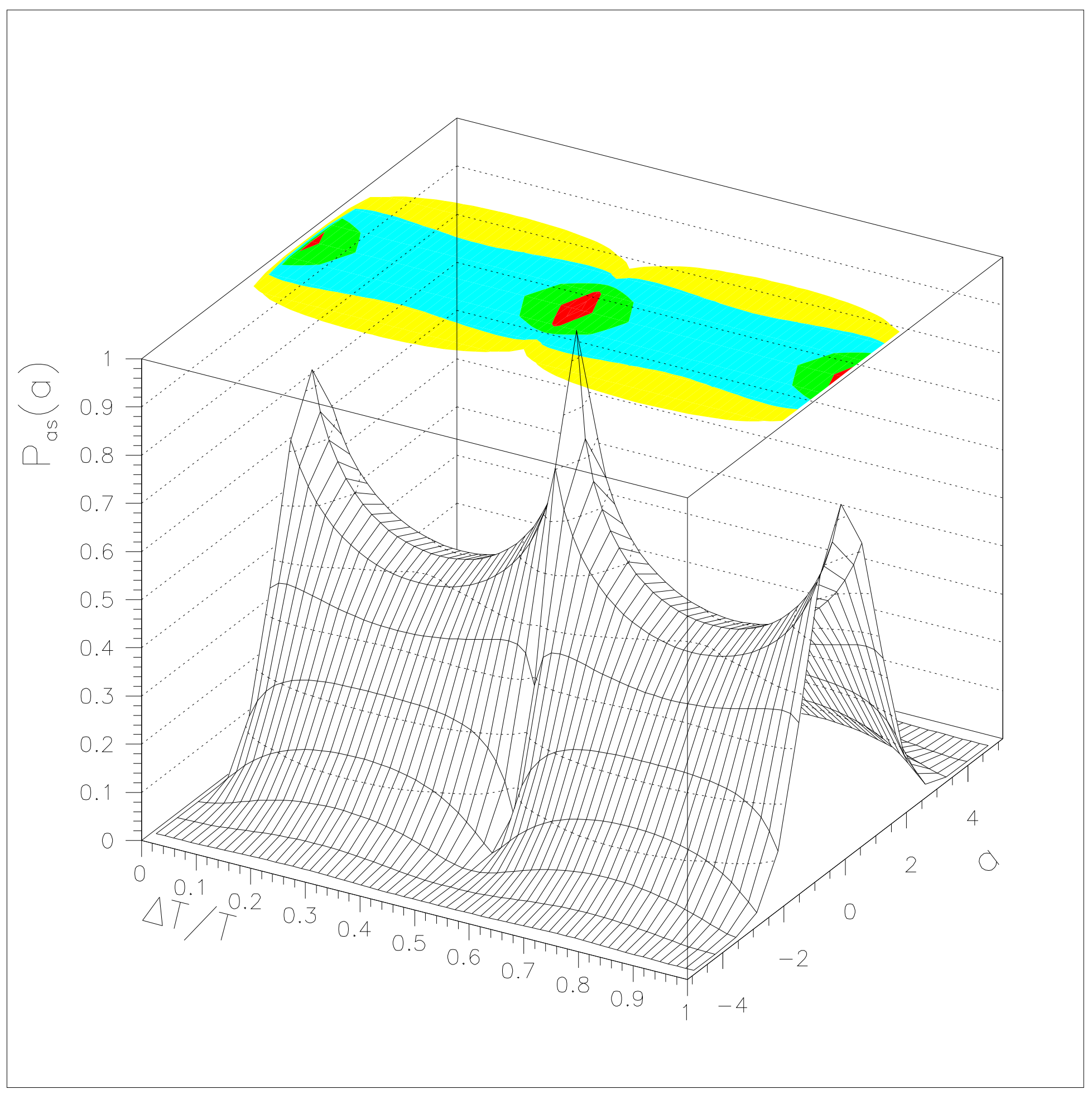




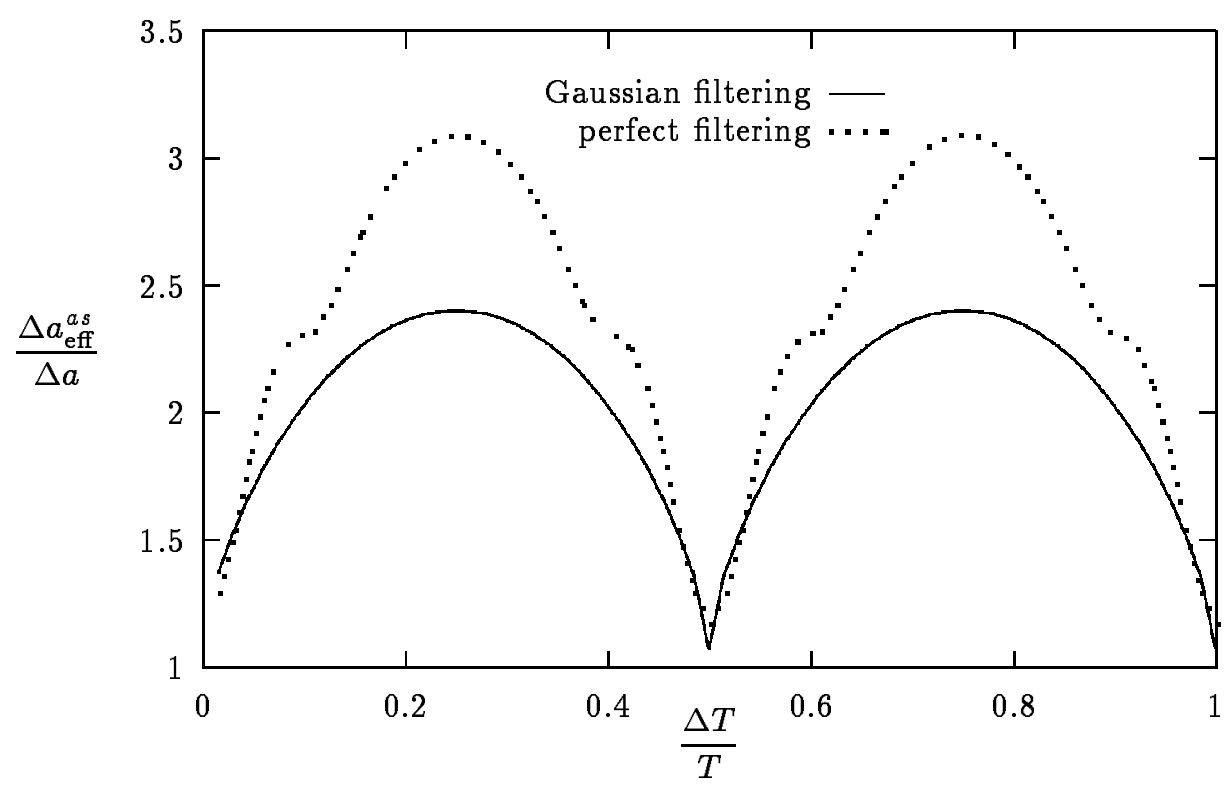

